\title{
A Discrete Hardy-Laptev-Weidl-Type Inequality and Associated Schrödinger-Type Operators
}

\author{
W. Desmond Evans and Karl Michael SCHMIDT
}

\author{
School of Mathematics \\ Cardiff University \\ Senghennydd Road \\ Cardiff CF24 4AG - Wales \\ EvansWD@cardiff.ac.uk schmidtkm@cardiff.ac.uk
}

Received: October 8, 2007

Accepted: April 7, 2008

\begin{abstract}
Although the classical Hardy inequality is valid only in the three- and higher dimensional case, Laptev and Weidl established a two-dimensional Hardy-type inequality for the magnetic gradient with an Aharonov-Bohm magnetic potential. Here we consider a discrete analogue, replacing the punctured plane with a radially exponential lattice. In addition to discrete Hardy and Sobolev inequalities, we study the spectral properties of two associated self-adjoint operators. In particular, it is shown that, for suitable potentials, the discrete Schrödingertype operator in the Aharonov-Bohm field has essential spectrum concentrated at 0 , and the multiplicity of its lower spectrum satisfies a CLR-type inequality.
\end{abstract}

Key words: discrete Schrödinger operator, Aharonov-Bohm magnetic potential. 2000 Mathematics Subject Classification: 47B37, 35P05, 39A70, 47A30, 81Q10.

\section{Introduction}

Hardy's well-known inequality

$$
\int_{\mathbb{R}^{n}} \frac{|f(\mathbf{x})|^{2}}{|\mathbf{x}|^{2}} d \mathbf{x} \leq\left|\frac{2}{n-2}\right|^{2} \int_{\mathbb{R}^{n}}|\nabla f(\mathbf{x})|^{2} d \mathbf{x}
$$


is valid for all $f \in C_{0}^{\infty}\left(\mathbb{R}^{n} \backslash\{0\}\right)$ and with the indicated constant best-possible: the inequality is trivial when $n=2$. For $n=2$, Laptev and Weidl [6] established a similar inequality, namely,

$$
\int_{\mathbb{R}^{2}} \frac{|f(\mathbf{x})|^{2}}{|\mathbf{x}|^{2}} d \mathbf{x} \leq C \int_{\mathbb{R}^{2}}\left|\nabla_{\mathbf{A}} f(\mathbf{x})\right|^{2} d \mathbf{x}
$$

in which $\nabla_{\mathbf{A}}$ is the magnetic gradient $\nabla_{\mathbf{A}}=\nabla+i \mathbf{A}$ and $\mathbf{A}$ is the magnetic potential of Aharonov-Bohm type given by

$$
\mathbf{A}(\mathbf{x})=\frac{\Psi(\theta)}{r}(-\sin \theta, \cos \theta),
$$

where $\mathbf{x}=(r \cos \theta, r \sin \theta)$ and $\Psi \in L^{\infty}(0,2 \pi)$. The best possible constant in (1) is

$$
C=\min _{k \in \mathbb{Z}}|\tilde{\Psi}-k|^{-2},
$$

where $\tilde{\Psi}$ is the magnetic flux

$$
\tilde{\Psi}:=\frac{1}{2 \pi} \int_{0}^{2 \pi} \Psi(\theta) d \theta
$$

Note that if $\tilde{\Psi} \in \mathbb{Z}$, there is no valid (i.e., non-trivial) inequality (1), since in this case it reduces, by gauge invariance, to Hardy's inequality, which is invalid in two dimensions.

Inequality (1) can be proved very simply by observing that the punctured plane $\mathbb{R}^{2} \backslash\{0\}$ is topologically equivalent to the cylinder $\mathbb{R} \times \mathbb{S}^{1}$. If we set $g(u, v)=e^{i \int_{0}^{v} \Psi} f\left(e^{u} \cos v, e^{u} \sin v\right)$ - which effects this topological transformation in conjunction with a gauge transformation — we find that (1) is equivalent to

$$
\int_{-\infty}^{\infty} \int_{0}^{2 \pi}|g(u, v)|^{2} d v d u \leq C \int_{-\infty}^{\infty} \int_{0}^{2 \pi}\left\{\left|\frac{\partial g}{\partial u}\right|^{2}+\left|\frac{\partial g}{\partial v}\right|^{2}\right\} d v d u
$$

where now $g \in C^{\infty}(\mathbb{R} \times[0,2 \pi])$ with compact support and

$$
g(u, 2 \pi)=e^{2 \pi i \tilde{\Psi}} g(u, 0), \quad \frac{\partial g}{\partial v}(u, 2 \pi)=e^{2 \pi i \tilde{\Psi}} \frac{\partial g}{\partial v}(u, 0) .
$$

The integral on the right-hand side of (4) is the quadratic form associated with a self-adjoint extension in $L^{2}(\mathbb{R} \times[0,2 \pi])$ of

$$
-\frac{\partial^{2}}{\partial u^{2}}-\frac{\partial^{2}}{\partial v^{2}}
$$

on $C_{0, \sharp}^{\infty}(\mathbb{R} \times[0,2 \pi])$, the set of functions in $C_{0}^{\infty}(\mathbb{R} \times[0,2 \pi])$ which satisfy the quasiperiodic boundary conditions (5). This self-adjoint operator has spectrum $\left[\min _{k \in \mathbb{Z}}|\tilde{\Psi}-k|^{2}, \infty\right)$, for, by separation of variables, it decouples into the non-negative 
self-adjoint operator $-\frac{d^{2}}{d u^{2}}$ in $L^{2}(\mathbb{R})$ with spectrum $[0, \infty)$ and the self-adjoint operator $-\frac{d^{2}}{d v^{2}}$ in $L^{2}([0,2 \pi])$ with quasi-periodic boundary conditions corresponding to (5), which has non-negative spectrum $\{\lambda \geq 0: \sqrt{\lambda}= \pm \tilde{\Psi} \bmod \mathbb{Z}\}$. Hence (1) and (3) follow directly.

However, the topological equivalence $\mathbb{R} \times \mathbb{S}^{1} \rightarrow \mathbb{R}^{2} \backslash\{0\},(u, v) \mapsto\left(e^{u} \cos v, e^{u} \sin v\right)$, transforms the weighted space $L^{2}\left(\mathbb{R} \times \mathbb{S}^{1} ; e^{2 u} d u d v\right)$ into $L^{2}\left(\mathbb{R}^{2}\right)$, and the self-adjoint operator in $L^{2}\left(\mathbb{R} \times \mathbb{S}^{1} ; e^{2 u} d u d v\right)$ associated with the right-hand side of (4) is the Friedrichs extension (see [4, VI. §2]) of

$$
-e^{-2 u}\left(\frac{\partial^{2}}{\partial u^{2}}+\frac{\partial^{2}}{\partial v^{2}}\right) .
$$

defined on the space of smooth functions of compact support. Thus, while inequality (4) (and hence (1)) is established by the analysis of the operator (6) as above, the operator (7) represents the magnetic Laplacian $-\nabla_{\mathbf{A}}^{2}:=-\nabla_{\mathbf{A}} \cdot \nabla_{\mathbf{A}}$ in the plane. A scaling argument shows that this operator has spectrum $[0, \infty)$.

In the present paper, we study a discrete variant of the Laptev-Weidl inequality as expressed in (4), and analyse the spectral properties of discrete analogues of the selfadjoint operators associated with (6) and (7). We discretise the cylinder $\mathbb{R} \times[0,2 \pi]$ to $\{j / m: j \in \mathbb{Z}\} \times\{2 \pi j / n: j \in\{1,2, \ldots, n\}\}:$ the corresponding point set in the physical plane accumulates at the origin and is exponentially sparse near infinity. On replacing $\partial g / \partial u$ and $\partial g / \partial v$ by $m\{g(j, k)-g(j-1, k)\}$ and $(n / 2 \pi)\{g(j, k)-g(j, k-1)\}$ respectively, the discrete analogue of (4) is

$$
\begin{aligned}
\frac{2 \pi}{m n} \sum_{j \in \mathbb{Z}} \sum_{k=1}^{n}|g(j, k)|^{2} \leq C \frac{2 \pi}{m n} \sum_{j \in \mathbb{Z}} \sum_{k=1}^{n}\left\{m^{2}|g(j, k)-g(j-1, k)|^{2}\right. & \\
& \left.+\left(\frac{n}{2 \pi}\right)^{2}|g(j, k)-g(j, k-1)|^{2}\right\} .
\end{aligned}
$$

For fixed $\tilde{\Psi} \in(0,1)$, we set $\delta:=e^{2 \pi i \tilde{\Psi}}$ and denote by $\mathcal{S}_{n}$ the set of quasiperiodic sequences

$$
\mathcal{S}_{n}:=\left\{(u(k))_{k \in \mathbb{Z}} \in \mathbb{C}^{\mathbb{Z}}: u(k+n)=\delta u(k) \quad(k \in \mathbb{Z})\right\},
$$

and by $\ell^{2}\left(\Lambda_{n}\right)$ the space $\mathcal{S}_{n}$ endowed with the norm induced by the inner product

$$
(u, v)_{\ell^{2}\left(\Lambda_{n}\right)}:=\sum_{k \in \Lambda_{n}} u(k) \overline{v(k)}
$$

where $\Lambda_{n}:=\{1,2, \ldots, n\}$. Furthermore, for positive weight $w(j)(j \in \mathbb{Z})$, denote by $\ell^{2}\left(\mathbb{Z} \times \Lambda_{n} ; w\right)$ the normed subspace of $\mathbb{C}^{\mathbb{Z}} \otimes \mathcal{S}_{n}$ (i.e., the space of complex sequences $(g(j, k))_{j, k \in \mathbb{Z}}$ that satisfy $\left.g(j, k+n)=\delta g(j, k), j, k \in \mathbb{Z}\right)$ with the inner product

$$
(g, h)_{\ell^{2}\left(\mathbb{Z} \times \Lambda_{n} ; w\right)}:=\sum_{j \in \mathbb{Z}} \sum_{k \in \Lambda_{n}} w(j) g(j, k) \overline{h(j, k)}
$$


we write $\ell^{2}\left(\mathbb{Z} \times \Lambda_{n}\right)$ for $\ell^{2}\left(\mathbb{Z} \times \Lambda_{n} ; 1\right)$.

We shall denote by $\Delta$ the left-difference operator $\Delta u(l):=u(l)-u(l-1)$, abbreviating

$$
\Delta^{2} u(l):=\Delta^{*} \Delta u(l)=u(l+1)+u(l-1)-2 u(l),
$$

and use the notation

$$
\Delta_{1} g(j, k):=g(j, k)-g(j-1, k), \quad \Delta_{2} g(j, k):=g(j, k)-g(j, k-1) .
$$

For $g \in \mathbb{C}^{\mathbb{Z}} \otimes \mathcal{S}_{n}$, the double sum on the right-hand side of (8) can easily be seen to be equal to

$$
-\sum_{j \in \mathbb{Z}} \sum_{k \in \Lambda_{n}}\left\{m^{2} \overline{g(j, k)} \Delta_{1}^{2} g(j, k)+\left(\frac{n}{2 \pi}\right)^{2} \overline{g(j, k)} \Delta_{2}^{2} g(j, k)\right\} .
$$

Hereafter we shall denote the bounded non-negative self-adjoint operator defined by $-\Delta^{2}$ on $\ell^{2}(\mathbb{Z})$ by $\mathcal{A}$ and that defined by $-\Delta^{2}$ on $\ell^{2}\left(\Lambda_{n}\right)$ by $\mathcal{B}_{n}$. (Note that $\mathcal{B}_{n}$ has a quasiperiodic 'boundary condition' $u(k+n)=\delta u(k), k \in \mathbb{Z}$.) The quadratic form (9) defines the following operator in $\ell^{2}\left(\mathbb{Z} \times \Lambda_{n}\right)$ :

$$
\left(\mathcal{D}_{m n} g\right)(j, k):=-m^{2} \Delta_{1}^{2} g(j, k)-\left(\frac{n}{2 \pi}\right)^{2} \Delta_{2}^{2} g(j, k) .
$$

On identifying $\ell^{2}\left(\mathbb{Z} \times \Lambda_{n}\right)$ with $\ell^{2}(\mathbb{Z}) \otimes \ell^{2}\left(\Lambda_{n}\right)$, we have that

$$
\mathcal{D}_{m n}=m^{2} \mathcal{A} \otimes 1+1 \otimes\left(\frac{n}{2 \pi}\right)^{2} \mathcal{B}_{n} .
$$

Thus $\mathcal{D}_{m n}$ is the discrete analogue of (6). In section 1 it will be shown to be a bounded operator on $\ell^{2}\left(\mathbb{Z} \times \Lambda_{n}\right)$ with a purely absolutely continuous spectrum, which we give explicitly. Moreover, we study the discrete analogue of (7),

$$
\mathcal{T}_{m n}^{0}=e^{-2 j / m} \mathcal{D}_{m n}=e^{-2 j / m}\left(m^{2} \mathcal{A} \otimes 1+1 \otimes\left(\frac{n}{2 \pi}\right)^{2} \mathcal{B}_{n}\right)
$$

acting in the weighted space $\ell^{2}\left(\mathbb{Z} \times \Lambda_{n} ; e^{2 j / m}\right)$. Its essential spectrum is $\{0\}$ (see Theorem 3.2 below).

Estimates for the numbers of eigenvalues of $\mathcal{D}_{m n}+V$ and of $\mathcal{T}_{m n}^{0}+V$ for suitable potentials $V: \mathbb{Z} \times \Lambda_{n} \rightarrow \mathbb{R}$ are determined in sections 2 and 3 , respectively.

\section{Discrete Hardy and Sobolev inequalities}

It is well known that the operator $\mathcal{A}$ has a purely absolutely continuous spectrum $[0,4]$; indeed, under the isometry of the Hilbert spaces $\ell^{2}(\mathbb{Z})$ and $L^{2}[0,2 \pi]$ generated by the latter's orthonormal basis $\left\{e^{i k x}(x \in[0,2 \pi]): k \in \mathbb{Z}\right\}$, the operator $\mathcal{A}$ is unitarily equivalent to the maximal operator of multiplication by the function $2(1-\cos )$ on $[0,2 \pi]$. The spectrum of $\mathcal{B}_{n}$ is the following discrete subset of $[0,4]$. 


\section{Lemma 1.1.}

$$
\sigma\left(\mathcal{B}_{n}\right)=\left\{\lambda_{l}: l \in\{0, \ldots, n-1\}\right\}
$$

where

$$
\lambda_{l}=4 \sin ^{2}\{(\tilde{\Psi}+l) \pi / n\} .
$$

These eigenvalues are all simple except for the case $\tilde{\Psi}=1 / 2$, where the eigenvalues are all double except for simple $\lambda_{\frac{n-1}{2}}$ if $n$ is odd.

Proof. The eigenvalue equation takes the form

$$
2 u(k)-u(k+1)-u(k-1)=\lambda u(k) \quad(k \in \mathbb{Z})
$$

with the additional condition $u(k+n)=\delta u(k)(k \in \mathbb{Z})$. This equation is equivalent to

$$
U(k+1)=M U(k) \quad(k \in \mathbb{Z})
$$

with $U(k):=\left(\begin{array}{c}u(k) \\ u(k-1)\end{array}\right)$ and $M:=\left(\begin{array}{cc}2-\lambda-1 \\ 1 & 0\end{array}\right)$, which clearly has the general solution $U(k)=M^{k} U(0)(k \in \mathbb{Z})$.

The boundary condition requires that $U(n)=\delta U(0)$. Hence $\lambda$ is an eigenvalue of $\mathcal{B}_{n}$ if and only if $\delta$ is an eigenvalue of $M^{n}$. The matrix $M$ has real trace and determinant 1 , and has non-real eigenvalues if and only if $\lambda \in(0,4)$. In this case, it has two complex conjugate eigenvalues of modulus 1 , namely

$$
\mu_{ \pm}=\frac{1}{2}(2-\lambda \pm \sqrt{\lambda(\lambda-4)})=: e^{ \pm i \phi} .
$$

Hence we require $e^{ \pm i n \phi}=\delta=e^{2 \pi i \tilde{\Psi}}$, or equivalently $\phi=\frac{2 \pi}{n}( \pm \tilde{\Psi}+l)$ with some $l \in \mathbb{Z}$. As $1-\lambda / 2=\operatorname{Re} \mu_{ \pm}=\cos \phi$, this yields

$$
\lambda=2\left(1-\cos \left(\frac{2 \pi}{n}(\tilde{\Psi}+l)\right)\right)=4 \sin ^{2}\left(\frac{\pi}{n}(\tilde{\Psi}+l)\right) .
$$

The assertion follows, as $\mathcal{B}_{n}$ has a discrete spectrum of multiplicity at most 2 .

Remark 1.2. By symmetry, we may, and shall hereafter, assume without loss of generality that $\tilde{\Psi} \in(0,1 / 2]$, and so we infer from Lemma 1.1 that

$$
\mathcal{B}_{n} \geq \min _{l \in\{0, \ldots, n-1\}} \lambda_{l}=\lambda_{0}=4 \sin ^{2}\left\{\frac{\pi}{n} \tilde{\Psi}\right\} .
$$

Similarly,

$$
\mathcal{B}_{n} \leq \max _{l \in\{0, \ldots, n-1\}} \lambda_{l}=\lambda_{\left[\frac{n}{2}\right]}=4 \sin ^{2}\left\{\frac{\pi}{n}\left(\tilde{\Psi}+\left[\frac{n}{2}\right]\right)\right\},
$$

with the Gauss bracket $[x]:=\max \{z \in \mathbb{Z}: z \leq x\}(x \in \mathbb{R})$. (Note that $\lambda_{0} \rightarrow 0$ while $\lambda_{\left[\frac{n}{2}\right]} \rightarrow 4$ as $n \rightarrow \infty$.) 
Since

$$
\sum_{k \in \Lambda_{n}} \overline{u(k)}\left(\mathcal{B}_{n} u\right)(k)=\sum_{k \in \Lambda_{n}}|u(k)-u(k-1)|^{2},
$$

we have established the inequalities

$$
C_{n} \frac{2 \pi}{n} \sum_{k=1}^{n}|u(k)|^{2} \leq \frac{n}{2 \pi} \sum_{k=1}^{n}|u(k)-u(k-1)|^{2} \leq \tilde{C}_{n} \frac{2 \pi}{n} \sum_{k=1}^{n}|u(k)|^{2}
$$

with the sharp constants

$$
C_{n}=\left(\frac{n}{\pi} \sin \left(\frac{\pi}{n} \tilde{\Psi}\right)\right)^{2}, \quad \tilde{C}_{n}=\left(\frac{n}{\pi} \sin \left(\frac{\pi}{n}\left(\tilde{\Psi}+\left[\frac{n}{2}\right]\right)\right)\right)^{2},
$$

for any sequence $(u(k))_{k \in \mathbb{Z}}$ with $u(k+n)=e^{2 \pi i \tilde{\Psi}} u(k)(k \in \mathbb{Z})$. It is of interest to note that $C_{n} \leq \tilde{\Psi}^{2}, \lim _{n \rightarrow \infty} C_{n}=\tilde{\Psi}^{2}$, and $\lim _{n \rightarrow \infty} \tilde{C}_{n}=\infty$.

As an immediate consequence of Lemma 1.1 and (10), we obtain

Lemma 1.3. The operator $\mathcal{D}_{m n}$ is bounded on $\ell^{2}\left(\mathbb{Z} \times \Lambda_{n}\right)$ and

$$
\sigma\left(\mathcal{D}_{m n}\right)=\left[\left(\frac{n}{2 \pi}\right)^{2} \lambda_{0}, 4 m^{2}+\left(\frac{n}{2 \pi}\right)^{2} \lambda_{\left[\frac{n}{2}\right]}\right],
$$

where $\lambda_{l}$ is given in (12). The spectrum of $\mathcal{D}_{m n}$ is purely absolutely continuous.

This lemma, together with observation (9), yields the following discrete analogue of the Laptev-Weidl inequality in $\mathbb{R}^{2}$.

Theorem 1.4. For all $g \in \ell^{2}\left(\mathbb{Z} \times \Lambda_{n}\right)$ and $\tilde{\Psi} \in(0,1 / 2]$,

$$
\begin{aligned}
\left\{\frac{n}{\pi} \sin \left(\frac{\pi}{n} \tilde{\Psi}\right)\right\}^{2} \sum_{j \in \mathbb{Z}} \sum_{k \in \Lambda_{n}}|g(j, k)|^{2} & \leq \mathfrak{d}_{m n}[g] \\
& \leq\left(4 m^{2}+\left\{\frac{n}{\pi} \sin \left(\frac{\pi}{n}\left(\tilde{\Psi}+\left[\frac{n}{2}\right]\right)\right)\right\}^{2}\right) \sum_{j \in \mathbb{Z}} \sum_{k \in \Lambda_{n}}|g(j, k)|^{2},
\end{aligned}
$$

where

$$
\mathfrak{d}_{m n}[g]:=\sum_{j \in \mathbb{Z}} \sum_{k \in \Lambda_{n}}\left\{m^{2}\left|\Delta_{1} g(j, k)\right|^{2}+\left(\frac{n}{2 \pi}\right)^{2}\left|\Delta_{2} g(j, k)\right|^{2}\right\} .
$$

Furthermore, we derive a discrete analogue of a Sobolev-type inequality for Schrödinger operators with Aharonov-Bohm magnetic potential (cf. [1, Theorem 1.1]).

Theorem 1.5. For all $g \in \ell^{2}\left(\mathbb{Z} \times \Lambda_{n}\right)$,

$$
\sup _{j \in \mathbb{Z}} \sum_{k \in \Lambda_{n}}|g(j, k)|^{2} \leq \frac{\pi}{2 m n \sin (\tilde{\Psi} \pi / n)} \mathfrak{d}_{m n}[g],
$$

where $\mathfrak{d}_{m n}$ is defined in (13). 
Proof. Let $\left\{u_{l}: l \in\{0, \ldots, n-1\}\right\}$ be the eigenvectors of $\mathcal{B}_{n}$ (orthonormal in $\ell^{2}\left(\Lambda_{n}\right)$ ) corresponding to the eigenvalues $\lambda_{l}$. Then

$$
g(j, k)=\sum_{l=0}^{n-1} c^{(l)}(j) u_{l}(k) \quad \text { with } \quad c^{(l)}(j)=\left(g(j, \cdot), u_{l}\right)_{\ell^{2}\left(\Lambda_{n}\right)},
$$

and we have

$$
\sum_{k \in \Lambda_{n}}\left|\Delta_{2} g(j, k)\right|^{2}=\sum_{k \in \Lambda_{n}} \overline{g(j, k)}\left[\mathcal{B}_{n} g(j, \cdot)\right](k)=\sum_{l=0}^{n-1} \lambda_{l}\left|c^{(l)}(j)\right|^{2} .
$$

Also

$$
\sum_{j \in \mathbb{Z}} \sum_{k \in \Lambda_{n}} \overline{g(j, k)}[\mathcal{A} g(\cdot, k)](j)=\sum_{j \in \mathbb{Z}} \sum_{k \in \Lambda_{n}}\left|\Delta_{1} g(j, k)\right|^{2}=\sum_{j \in \mathbb{Z}} \sum_{l=0}^{n-1}\left|\Delta c^{(l)}(j)\right|^{2} .
$$

Thus

$$
\mathfrak{d}_{m n}[g]=\sum_{j \in \mathbb{Z}} \sum_{l=0}^{n-1}\left\{m^{2}\left|\Delta c^{(l)}(j)\right|^{2}+\left(\frac{n}{2 \pi}\right)^{2} \lambda_{l}\left|c^{(l)}(j)\right|^{2}\right\} .
$$

Since $c^{(l)}(j) \rightarrow 0$ as $j \rightarrow \pm \infty$, we have, with

$$
\varepsilon_{t j}:=\left\{\begin{array}{ll}
1, & t \leq j, \\
-1, & t \geq j+1
\end{array} \quad(t \in \mathbb{Z})\right.
$$

that

$$
\begin{aligned}
\left|c^{(l)}(j)\right|^{2} & =\frac{1}{2}\left(\sum_{t \leq j}\left(\left|c^{(l)}(t)\right|^{2}-\left|c^{(l)}(t-1)\right|^{2}\right)-\sum_{t \geq j+1}\left(\left|c^{(l)}(t)\right|^{2}-\left|c^{(l)}(t-1)\right|^{2}\right)\right) \\
& =\frac{1}{2} \sum_{t \in \mathbb{Z}} \varepsilon_{t j}\left(\overline{c^{(l)}(t)} \Delta c^{(l)}(t)+c^{(l)}(t-1) \overline{\Delta c^{(l)}(t)}\right) \\
& \leq\left(\sum_{t \in \mathbb{Z}}\left|c^{(l)}(t)\right|^{2}\right)^{1 / 2}\left(\sum_{t \in \mathbb{Z}}\left|\Delta c^{(l)}(t)\right|^{2}\right)^{1 / 2} \\
& =\left(\frac{2 \pi}{m n}\right) \lambda_{0}^{-1 / 2}\left(\sum_{t \in \mathbb{Z}}\left(\frac{n}{2 \pi}\right)^{2} \lambda_{0}\left|c^{(l)}(t)\right|^{2}\right)^{1 / 2}\left(\sum_{t \in \mathbb{Z}} m^{2}\left|\Delta c^{(l)}(t)\right|^{2}\right)^{1 / 2} \\
& \leq \frac{\pi}{m n} \lambda_{0}^{-1 / 2} \sum_{t \in \mathbb{Z}}\left(m^{2}\left|\Delta c^{(l)}(t)\right|^{2}+\left(\frac{n}{2 \pi}\right)^{2} \lambda_{0}\left|c^{(l)}(t)\right|^{2}\right) .
\end{aligned}
$$

In view of (14), this gives

$$
\sum_{k \in \Lambda_{n}}|g(j, k)|^{2}=\sum_{l=0}^{n-1}\left|c^{(l)}(j)\right|^{2} \leq \frac{\pi}{m n} \lambda_{0}^{-1 / 2} \mathfrak{d}_{m n}[g]=\frac{\pi}{2 m n \sin (\tilde{\Psi} \pi / n)} \mathfrak{d}_{m n}[g]
$$

for any $j \in \mathbb{Z}$, whence the result. 


\section{The lower spectrum}

It is well-known that the Cwikel-Lieb-Rosenblum (CLR) inequality (see $[2,7,8]$ )

$$
N\left(-\nabla^{2}+V\right) \leq \mathrm{const} \int_{\mathbb{R}^{n}} V_{-}^{n / 2} d x, \quad V_{-}:=-\min \{0, V\}, \quad n \geq 3,
$$

for the number of negative eigenvalues of the Schrödinger operator defined by $-\nabla^{2}+V$ with $V \in L^{n / 2}\left(\mathbb{R}^{n}\right)$ is not valid for $n=2$. In [1], a modified inequality was derived for $N\left(-\nabla_{\mathbf{A}}^{2}+V\right)$ for the $\mathbf{A}$ in $(2), \tilde{\Psi} \notin \mathbb{Z}$ and $V \in L^{1}\left(\mathbb{R}^{+} ; L^{\infty}, r d r\right)$. In the next section, we shall obtain an analogous inequality for the discrete operator $\mathcal{T}_{m n}^{0}+V$, which, as seen in the introduction, corresponds to the Aharonov-Bohm Schrödinger operator in the plane. We first consider, in the present section, the question of estimating the number $N\left(\mathcal{D}_{m n}+V\right)$ for suitable potentials $V$.

As a central ingredient, we use the following preliminary lemma, which shows that for non-negative operators $A$ in an $\ell^{2}(\mathbb{Z} ; w)$ space, the number of negative eigenvalues of $A+W$ is bounded by the number of negative values of the potential $W: \mathbb{Z} \rightarrow \mathbb{R}$. We then also observe that in the case $A=\mathcal{A}, w=1$, this estimate is sharp, and there is no estimate in terms of the overall size of $W_{-}$.

Lemma 2.1. Let $A$ be a non-negative self-adjoint operator in $\ell^{2}(\mathbb{Z} ; w)$ and $W$ the multiplication operator induced by a real-valued sequence $(W(j))_{j \in \mathbb{Z}}$. Assume that the quadratic form of $A+W$ is bounded below, and let $A+W$ be the self-adjoint operator defined as a form sum. Then the number of negative eigenvalues of $A+W$ is not larger than $\sharp\{j: W(j)<0\}$.

Proof. Let $v_{1}, v_{2}, \ldots, v_{N}$ be the orthonormal eigenvectors of $A+W$ corresponding to its negative eigenvalues $\lambda_{m}, m \in\{1,2, \ldots, N\}$, and suppose that $M:=\{j \in \mathbb{Z}: W(j)<0\}$ has less than $N$ elements. Then the vectors $\tilde{v}_{m}:=\left(v_{m}(j)\right)_{j \in M} \in \mathbb{C}^{|M|}, m=1,2, \ldots, N$, must be linearly dependent and so there are numbers $\alpha_{m} \in \mathbb{C}, m=1,2, \ldots, N$, not all zero, such that

$$
\sum_{m=1}^{N} \alpha_{m} \tilde{v}_{m}=0
$$

Let $u:=\sum_{m=1}^{N} \alpha_{m} v_{m}$; then $u \in Q(A+W)$, the form domain of $A+W$, because every eigenvector lies in the form domain. Moreover, $u(j)=0$ for all $j \in M$ and hence

$$
\begin{aligned}
0 & \leq \sum_{m \in \mathbb{Z} \backslash M} w(j) W(j)|u(j)|^{2}=\sum_{m \in \mathbb{Z}} w(j) W(j)|u(j)|^{2}=(W u, u)_{\ell^{2}(\mathbb{Z} ; w)} \\
& \leq((A+W) u, u)_{\ell^{2}(\mathbb{Z} ; w)}=\sum_{m=1}^{N} \lambda_{m}\left|\alpha_{m}\right|^{2}<0,
\end{aligned}
$$

a contradiction. 
In this section, we are particularly interested in the case $A=\mathcal{A}$; the estimate given in Lemma 2.1 is then best possible in the following sense.

Lemma 2.2. For any $\varepsilon>0$ and $N \in \mathbb{N}$, there is a real-valued potential $W$ which has only $N$ negative values, each of absolute value less than $\varepsilon$, such that $\mathcal{A}+W$ has $N$ negative eigenvalues.

Proof. Consider the potential $W$ with $W(j)=0(j \neq 0)$ and $-\varepsilon<W(0)<0$. For an integer $K>2 /|W(0)|$, define

$$
v_{m}:= \begin{cases}1-|m| / K, & \text { if }|m| \leq K \\ 0, & \text { if }|m|>K\end{cases}
$$

Then

$$
(\mathcal{A} v, v)_{\ell^{2}(\mathbb{Z})}=\sum_{m \in \mathbb{Z}}\left|v_{m}-v_{m-1}\right|^{2}=2 \sum_{m=1}^{K}|(1-m / K)-(1-(m-1) / K)|^{2} \leq 2 / K .
$$

Hence $((\mathcal{A}+W) v, v)_{\ell^{2}(\mathbb{Z})} \leq 2 / K+W(0)<0$.

Placing $N$ shifted copies of $W$ at least $2 K+2$ apart, we obtain a potential with the property that the quadratic form of $\mathcal{A}+W$ is negative on a space of dimension $N$. It follows by the min-max principle that $\mathcal{A}+W$ has $N$ negative eigenvalues.

In the following, we denote by $N(\mathcal{T} ; \mu)$ the number of eigenvalues of a self-adjoint operator $\mathcal{T}$ below $\mu$, where $\mu \leq \inf \sigma_{e}(\mathcal{T})$. Moreover, let $Y:=\ell^{1}\left(\mathbb{Z} ; \ell^{\infty}\left(\Lambda_{n}\right)\right)$ with norm

$$
\|V\|_{Y}:=\sum_{j \in \mathbb{Z}} \max _{k \in \Lambda_{n}}|V(j, k)| .
$$

Theorem 2.3. Let $(V(j, k))_{j \in \mathbb{Z}, k \in \Lambda_{n}}$ be real-valued with $\lim _{j \rightarrow \pm \infty} V(j, k)=0$ and $V_{-} \in Y$, and let $\tilde{\Psi} \in(0,1 / 2]$. Then $\mathcal{D}_{m n}+V$ is a bounded self-adjoint operator on $\ell^{2}\left(\mathbb{Z} \times \Lambda_{n}\right)$ and has the following properties:

(i) $\sigma_{\text {ess }}\left(\mathcal{D}_{m n}+V\right)=\left[\left(\frac{n}{2 \pi}\right)^{2} \lambda_{0}, 4 m^{2}+\left(\frac{n}{2 \pi}\right)^{2} \lambda_{\left[\frac{n}{2}\right]}\right]$.

(ii) For any $\mu<\left(\frac{n}{2 \pi}\right)^{2} \lambda_{0}$,

$$
N\left(\mathcal{D}_{m n}+V ; \mu\right) \leq \sum_{l=0}^{n-1}\left[\left(\left(\frac{n}{2 \pi}\right)^{2} \lambda_{l}-\mu\right)^{-1}\left\|V_{-}\right\|_{Y}\right],
$$

where [.] is again the Gauss bracket.

(iii) There does not exist an estimate of the form

$$
N\left(\mathcal{D}_{m n}+V ;\left(\frac{n}{2 \pi}\right)^{2} \lambda_{0}\right) \leq K\left\|V_{-}\right\|_{Y}
$$

for all $V \in Y$. 
Remark 2.4. In particular, the inequality (15) with $\mu=0$ gives an estimate for the number of negative eigenvalues of $\mathcal{D}_{m n}+V$. Note that (15) implies the slightly worse estimate

$$
N\left(\mathcal{D}_{m n}+V ; \mu\right) \leq K(\mu)\left\|V_{-}\right\|_{Y} \quad \text { with } \quad K(\mu)=\sum_{l=1}^{n-1}\left(\left(\frac{n}{2 \pi}\right)^{2} \lambda_{l}-\mu\right)^{-1} .
$$

Proof. (i) $V$ generates a bounded self-adjoint multiplication operator in $\ell^{2}\left(\mathbb{Z} \times \Lambda_{n}\right)$ with operator norm $\|V\|=\max _{(j, k) \in \mathbb{Z} \times \Lambda_{n}}|V(j, k)|$.

Let $\varepsilon>0$; then

$$
V_{\varepsilon}(j, k):= \begin{cases}V(j, k) & \text { if }|V(j, k)|>\varepsilon \\ 0 & \text { otherwise }\end{cases}
$$

has a finite support and hence generates a finite-rank multiplication operator with $\left\|V-V_{\varepsilon}\right\| \leq \varepsilon$ (in operator norm). Thus $V$ is a norm limit of finite-rank operators, so it is compact and (i) follows by Weyl's perturbation theorem.

(ii) Let $W(j):=-\left\|V_{-}(j, \cdot)\right\|_{\ell^{\infty}\left(\Lambda_{n}\right)} \leq 0$. Then $\|W\|_{\ell^{1}(\mathbb{Z})}=\left\|V_{-}\right\|_{Y}$ and $\mathcal{D}_{m n}+W \leq \mathcal{D}_{m n}+V$, so that $N\left(\mathcal{D}_{m n}+V ; \mu\right) \leq N\left(\mathcal{D}_{m n}+W ; \mu\right)$. Moreover, if $\left\{u_{l}: l \in\{0, \ldots, n-1\}\right\}$ are the orthonormal eigenvectors of $\mathcal{B}_{n}$ in $\ell^{2}\left(\Lambda_{n}\right)$, we have

$$
\ell^{2}\left(\mathbb{Z} \times \Lambda_{n}\right) \cong \ell^{2}(\mathbb{Z}) \otimes \ell^{2}\left(\Lambda_{n}\right)=\bigoplus_{l=0}^{n-1}\left(\ell^{2}(\mathbb{Z}) \otimes\left[u_{l}\right]\right),
$$

where [·] denotes the linear span, and

$$
\mathcal{D}_{m n}+W=\bigoplus_{l=0}^{n-1}\left(m^{2} \mathcal{A}+\left(\frac{n}{2 \pi}\right)^{2} \lambda_{l}+W\right) \otimes 1
$$

where 1 denotes the identity on $\left[u_{l}\right]$.

We therefore conclude from Lemma 2.1 that

$$
N\left(\mathcal{D}_{m n}+W ; \mu\right)=\sum_{l=0}^{n-1} N\left(m^{2} \mathcal{A}+\left(\frac{n}{2 \pi}\right)^{2} \lambda_{l}+W-\mu ; 0\right) \leq \sum_{l=0}^{n-1} M_{l},
$$

where

Now

$$
M_{l}:=\sharp\left\{j \in \mathbb{Z}: W(j)<\mu-\left(\frac{n}{2 \pi}\right)^{2} \lambda_{l}\right\} .
$$

$$
\left\|V_{-}\right\|_{Y}=\|W\|_{\ell^{1}(\mathbb{Z})}=-\sum_{j \in \mathbb{Z}} W(j)>M_{l}\left(\left(\frac{n}{2 \pi}\right) \lambda_{l}-\mu\right)
$$

for each $l \in\{0, \ldots, n-1\}$, and (15) follows.

(iii) We use Lemma 2.2. Given $\varepsilon>0$ and $N \in \mathbb{N}$, there exists a potential $W \in \ell^{1}(\mathbb{Z})$ such that $m^{2} \mathcal{A}+\left(\frac{n}{2 \pi}\right)^{2} \lambda_{1}+W$ has $N$ eigenvalues below $\left(\frac{n}{2 \pi}\right)^{2} \lambda_{1}$ and $\|W\|_{\ell^{1}(\mathbb{Z})}<\varepsilon$. Then $V(j, k):=W(j)$ will have the same property for $\mathcal{D}_{m n}+V$ and $\|V\|_{Y}<\varepsilon$. Since $\varepsilon$ is arbitrarily small, the assertion follows. 


\section{A discrete CLR-type inequality}

As noted in the introduction, $\mathcal{D}_{m n}$ is not the analogue of the magnetic Laplacian, as the transformation of the punctured plane to logarithmic polar coordinates introduces an exponential weight in the radial variable (see (7)), and this is reflected in the operator $\mathcal{T}_{m n}^{0}$ of (11). Thus the discrete analogue of the magnetic Schrödinger operator $-\nabla_{A}^{2}+V$ is

$$
\mathcal{T}_{m n}^{0}+V=e^{-2 j / m}\left(m^{2} \mathcal{A} \otimes 1+1 \otimes\left(\frac{n}{2 \pi}\right)^{2} \mathcal{B}_{n}\right)+V
$$

acting in $\ell^{2}\left(\mathbb{Z} \times \Lambda_{n} ; e^{2 j / m}\right)$.

We consider potentials $V: \mathbb{Z} \times \Lambda_{n} \rightarrow \mathbb{R}$ satisfying the hypothesis

$$
\lim _{j \rightarrow-\infty} V(j, k) e^{2 j / m}=0, \quad \lim _{j \rightarrow \infty} V(j, k)=0 \quad \text { for all } k \in \Lambda_{n} .
$$

Note that (H1) admits certain unbounded potentials, e.g., $V(j, k)=-c e^{-j / m}$ $\left(j \in \mathbb{Z}, k \in \Lambda_{n}\right)$ with constant $c$, which corresponds to the physical Coulomb potential $\frac{-c}{|\mathbf{x}|}$ after the topological transformation to the cylinder and discretisation outlined in the introduction.

Under assumption (H1), the quadratic form for the above operator is bounded below, as shown in the following lemma, and hence there is a corresponding selfadjoint operator $\mathcal{T}_{m n}$ in $\ell^{2}\left(\mathbb{Z} \times \Lambda_{n} ; e^{2 j / m}\right)$ associated with the closure of the quadratic form defined for sequences which are finite in the $j$-direction.

Lemma 3.1. Under hypothesis (H1), there is a constant $M \in \mathbb{R}$ such that

$$
\begin{aligned}
&\left(\left(e^{-2 j / m}\left(m^{2} \mathcal{A} \otimes 1+1 \otimes\left(\frac{n}{2 \pi}\right)^{2} \mathcal{B}_{n}\right)+V\right) u, u\right)_{\ell^{2}\left(\mathbb{Z} \times \Lambda_{n} ; e^{2 j / m}\right)} \\
& \geq M\|u\|_{\ell^{2}\left(\mathbb{Z} \times \Lambda_{n} ; e^{2 j / m}\right)}^{2}
\end{aligned}
$$

for all $u \in \ell^{2}\left(\mathbb{Z} \times \Lambda_{n} ; e^{2 j / m}\right)$.

Proof. For each $u \in \ell^{2}\left(\mathbb{Z} \times \Lambda_{n} ; e^{2 j / m}\right)$ such that the left-hand side of (16) is finite, we have

$$
\begin{aligned}
\left(\left(e^{-2 j / m}\right.\right. & \left.\left.\left(m^{2} \mathcal{A} \otimes 1+1 \otimes\left(\frac{n}{2 \pi}\right)^{2} \mathcal{B}_{n}\right)+V\right) u, u\right)_{\ell^{2}\left(\mathbb{Z} \times \Lambda_{n} ; e^{2 j / m}\right)} \\
= & \sum_{j \in \mathbb{Z}}\left(\sum_{k \in \Lambda_{n}} m^{2}|u(j, k)-u(j-1, k)|^{2}+\left(\frac{n}{2 \pi}\right)^{2} \sum_{k \in \Lambda_{n}} 1 \otimes \mathcal{B}_{n} u(j, k) \overline{u(j, k)}\right. \\
& \left.+\sum_{k \in \Lambda_{n}} V(j, k)|u(j, k)|^{2} e^{2 j / m}\right) \\
\geq & \sum_{j \in \mathbb{Z}} \sum_{k \in \Lambda_{n}}\left(\left(\frac{n}{2 \pi}\right)^{2} \lambda_{0}+V(j, k) e^{2 j / m}\right) e^{-2 j / m}|u(j, k)|^{2} e^{2 j / m} .
\end{aligned}
$$


As $\left(\left(\frac{n}{2 \pi}\right)^{2} \lambda_{0}+V(j, k) e^{2 j / m}\right) e^{-2 j / m}$ tends to $\infty$ as $j \rightarrow-\infty$ and to 0 as $j \rightarrow \infty$, it is bounded below.

The essential spectrum of the self-adjoint operator $\mathcal{T}_{m n}$ can be characterised as follows.

Theorem 3.2. Under hypothesis $(\mathrm{H} 1), \sigma_{\mathrm{ess}}\left(\mathcal{T}_{m n}\right)=\{0\}$.

Proof. Let $\lambda \in \sigma_{\text {ess }}\left(\mathcal{T}_{m n}\right)$. Then there is a singular sequence $\left(u_{i}\right)_{i \in \mathbb{N}}$, i.e., such that $\liminf _{i \rightarrow \infty}\left\|u_{i}\right\|_{\ell^{2}\left(\mathbb{Z} \times \Lambda_{n} ; e^{2 j / m}\right)} \geq 1, \lim _{i \rightarrow \infty}\left\|\left(\mathcal{T}_{m n}-\lambda\right) u_{i}\right\|_{\ell^{2}\left(\mathbb{Z} \times \Lambda_{n} ; e^{2 j / m}\right)}=0$, and $u_{i} \rightarrow 0$ $(i \rightarrow \infty)$ in $\ell^{2}\left(\mathbb{Z} \times \Lambda_{n} ; e^{2 j / m}\right)$.

Now let $l \in \mathbb{N}$. For $J \in\{-l, \ldots, l\}$ and each $k \in \Lambda_{n}$,

$$
u_{i}(J, k)=\left(u_{i}, \chi_{\{(J, k)\}}\right)_{\ell^{2}\left(\mathbb{Z} \times \Lambda_{n} ; e^{2 j / m}\right)} e^{-2 J / m} \longrightarrow 0 \quad(i \rightarrow \infty)
$$

where $\chi_{\{(J, k)\}}$ is the characteristic function of $\{(J, k)\}$, so $\chi_{\{-l, \ldots, l\} \times \Lambda_{n}} u_{i} \rightarrow 0$ and similarly $\chi_{\{-l, \ldots, l\} \times \Lambda_{n}}(\mathcal{A} \otimes 1) u_{i} \rightarrow 0(i \rightarrow \infty)$. Thus we can select a subsequence $\left(\tilde{u}_{l}\right)_{l \in \mathbb{N}}$ of $\left(u_{i}\right)_{i \in \mathbb{N}}$ such that

$$
\left\|\chi_{\{-l, \ldots, l\} \times \Lambda_{n}} \tilde{u}_{l}\right\|_{\ell^{2}\left(\mathbb{Z} \times \Lambda_{n} ; e^{2 j / m}\right)},\left\|\chi_{\{-l-1, \ldots, l+1\} \times \Lambda_{n}}\left(\mathcal{T}_{m n}-\lambda\right) \tilde{u}_{l}\right\|_{\ell^{2}\left(\mathbb{Z} \times \Lambda_{n} ; e^{2 j / m}\right)}<\frac{1}{l} .
$$

Then $v_{l}:=\left(1-\chi_{\{-l, \ldots, l\} \times \Lambda_{n}}\right) \tilde{u}_{l}(l \in \mathbb{N})$ will form a singular sequence for $\mathcal{T}_{m n}-\lambda$ with the additional property that $v_{l}(j, k)=0(j \in\{-l, \ldots, l\})$. We can write $v_{l}=v_{l}^{+}+v_{l}^{-}$, where $\operatorname{supp} v_{l}^{ \pm} \subset \pm\{l, \ldots\} \times \Lambda_{n}$; then by orthogonality

$$
\begin{aligned}
& \left\|v_{l}\right\|_{\ell^{2}\left(\mathbb{Z} \times \Lambda_{n} ; e^{2 j / m}\right)}^{2}=\left\|v_{l}^{+}\right\|_{\ell^{2}\left(\mathbb{Z} \times \Lambda_{n} ; e^{2 j / m}\right)}^{2}+\left\|v_{l}^{-}\right\|_{\ell^{2}\left(\mathbb{Z} \times \Lambda_{n} ; e^{2 j / m}\right)}^{2}, \\
& \left\|\left(\mathcal{T}_{m n}-\lambda\right) v_{l}\right\|_{\ell^{2}\left(\mathbb{Z} \times \Lambda_{n} ; e^{2 j / m}\right)}^{2} \\
& \quad=\left\|\left(\mathcal{T}_{m n}-\lambda\right) v_{l}^{+}\right\|_{\ell^{2}\left(\mathbb{Z} \times \Lambda_{n} ; e^{2 j / m}\right)}^{2}+\left\|\left(\mathcal{T}_{m n}-\lambda\right) v_{l}^{-}\right\|_{\ell^{2}\left(\mathbb{Z} \times \Lambda_{n} ; e^{2 j / m}\right.}^{2} .
\end{aligned}
$$

Now we prove that $\left\|v_{l}^{-}\right\|_{\ell^{2}\left(\mathbb{Z} \times \Lambda_{n} ; e^{2 j / m}\right)} \rightarrow 0$. Suppose that this is not the case; then $\left(v_{l}^{-}\right)_{l \in \mathbb{N}}$ is a singular sequence for $\mathcal{T}_{m n}-\lambda$. Moreover, if we denote by $\mathcal{T}_{m n}^{K,-}$ the self-adjoint operator on the subspace

$$
\mathcal{H}^{K,-}=\left\{g \in \ell^{2}\left(\mathbb{Z} \times \Lambda_{n} ; e^{2 j / m}\right): g(j, k)=0 \quad\left(j>-K, \quad k \in \Lambda_{n}\right)\right\}
$$

defined by restriction of the quadratic form for $\mathcal{T}_{m n}$ to this subspace, $\left(v_{l}^{-}\right)_{l>K}$ will be a singular sequence for $\mathcal{T}_{m n}^{K,-}-\lambda$, so $\lambda \in \sigma\left(\mathcal{T}_{m n}^{K,-}\right)$ for any $K \in \mathbb{N}$. 
On the other hand, for any $u \in Q\left(\mathcal{T}_{m n}^{K,-}\right)$ (the form domain) we have

$$
\begin{aligned}
\left(\mathcal{T}_{m n}^{K,-} u, u\right)_{\mathcal{H}^{K,-}}= & \sum_{k \in \Lambda_{n}} \sum_{j \leq-K}\left(m^{2}\left|\Delta_{1} u(j, k)\right|^{2}+\left(\frac{n}{2 \pi}\right)^{2}\left(1 \otimes \mathcal{B}_{n} u(j, k)\right) \overline{u(j, k)}\right. \\
& \left.+V(j, k) e^{2 j / m}|u(j, k)|^{2}\right) \\
\geq & \sum_{k \in \Lambda_{n}} \sum_{j \leq-K}\left(\left(\frac{n}{2 \pi}\right)^{2} \lambda_{0}+V(j, k) e^{2 j / m}\right) e^{-2 j / m}|u(j, k)|^{2} e^{2 j / m} \\
\geq & \left(\left(\frac{n}{2 \pi}\right)^{2} \lambda_{0}-\sup _{k \in \Lambda_{n}} \sup _{j \leq-K}\left|e^{2 j / m} V(j, k)\right|\right) e^{2 K / m}\|u\|_{\mathcal{H}^{K,-}}^{2},
\end{aligned}
$$

so the Rayleigh quotient will be greater than $\lambda$ for sufficiently large $K$, contradicting $\lambda \in \sigma\left(\mathcal{T}_{m n}^{K,-}\right)$.

Consequently, $\left(v_{l}^{+}\right)_{l \in \mathbb{N}}$ is a singular sequence for $\mathcal{T}_{m n}-\lambda$. Note that $v_{l}^{+} \in$ $\ell^{2}\left(\mathbb{Z} \times \Lambda_{n}\right)$ as well. Using Lemma 1.3 and the second requirement in (H1), we obtain

$$
\begin{aligned}
\| \mathcal{T}_{m n} & v_{l}^{+} \|_{\ell^{2}\left(\mathbb{Z} \times \Lambda_{n} ; e^{2 j / m}\right)}^{2} \\
& =\sum_{j \in \mathbb{Z}} \sum_{k \in \Lambda_{n}}\left|\left(e^{-2 j / m} \mathcal{D}_{m n}+V\right) v_{l}^{+}(j, k)\right|^{2} e^{2 j / m} \\
& \leq 2 e^{-2 l / m} \sum_{j \geq l} \sum_{k \in \Lambda_{n}}\left|\mathcal{D}_{m n} v_{l}^{+}(j, k)\right|^{2}+2 \sup _{\{l, \ldots\} \times \Lambda_{n}}|V|^{2} \sum_{j \geq l} \sum_{k \in \Lambda_{n}}\left|v_{l}^{+}(j, k)\right|^{2} e^{2 j / m} \\
& \leq 2\left(e^{-2 l / m}\left(4 m^{2}+\left(\frac{n}{2 \pi}\right)^{2} \lambda_{\left[\frac{n}{2}\right]}\right)^{2}+\sup _{\{l, \ldots\} \times \Lambda_{n}}|V|^{2}\right)\left\|v_{l}^{+}\right\|_{\ell^{2}\left(\mathbb{Z} \times \Lambda_{n} ; e^{2 j / m}\right)}^{2} \\
& \longrightarrow 0 \quad(l \rightarrow \infty),
\end{aligned}
$$

so $\lambda=0$ follows.

To show that 0 is indeed a point of the essential spectrum, consider the weak null sequence $\left(u_{i}\right)_{i \in \mathbb{N}}$, where $u_{i}(j, k)=e^{-i / m} \delta_{i j} \delta_{0 k}\left(j \in \mathbb{Z}, k \in \Lambda_{n}\right)$, for which

$$
\left(\mathcal{T}_{m n} u_{i}, u_{i}\right)_{\ell^{2}\left(\mathbb{Z} \times \Lambda_{n} ; e^{2 j / m}\right)}=2\left(m^{2}+\left(\frac{n}{2 \pi}\right)^{2}\right) e^{-2 i / m}+V(i, 0) \longrightarrow 0 \quad(i \rightarrow \infty) .
$$

The analogue of Theorem 2.3 is

Theorem 3.3. Assume that $V$ satisfies hypothesis (H1), and that in addition $V_{-} \in \tilde{Y}:=\ell^{1}\left(\mathbb{Z}, \ell^{\infty}\left(\Lambda_{n}\right) ; e^{2 j / m}\right)$. Then, with the operator $\mathcal{T}_{m n}$ defined as above,

$$
N\left(\mathcal{T}_{m n} ; 0\right) \leq \sum_{l=0}^{n-1}\left[\frac{1}{\left(\frac{n}{2 \pi}\right)^{2} \lambda_{l}}\left\|V_{-}\right\|_{\tilde{Y}}\right]
$$


Proof. Let $W(j):=-\left\|V_{-}(j, \cdot)\right\|_{\ell^{\infty}\left(\Lambda_{n}\right)}$ and $W$ the corresponding multiplication operator. Then, for $\varphi \in Q\left(\mathcal{T}_{m n}\right)$, the form domain of $\mathcal{T}_{m n}$, and denoting by $\tilde{\mathcal{T}}_{m n}$ the operator with potential $W$,

$$
\begin{aligned}
\left(\mathcal{T}_{m n} \varphi, \varphi\right)_{\ell^{2}\left(\mathbb{Z} \times \Lambda_{n}, e^{2 j / m}\right) \geq} & \left(\tilde{\mathcal{T}}_{m n} \varphi, \varphi\right)_{\ell^{2}\left(\mathbb{Z} \times \Lambda_{n}, e^{2 j / m}\right)} \\
= & \sum_{j \in \mathbb{Z}} \sum_{k \in \Lambda_{n}}\left(m^{2}\left|\Delta_{1} \varphi(j, k)\right|^{2}+\left(\frac{n}{2 \pi}\right)^{2}\left|\Delta_{2} \varphi(j, k)\right|^{2}\right. \\
& \left.+W(j)|\varphi(j, k)|^{2} e^{2 j / m}\right) \\
= & \sum_{j \in \mathbb{Z}} \sum_{l=0}^{n-1}\left(m^{2}\left|\Delta c^{(l)}(j)\right|^{2}+\left(\frac{n}{2 \pi}\right)^{2} \lambda_{l}\left|c^{(l)}(j)\right|^{2}\right. \\
& \left.+W(j) e^{2 j / m}\left|c^{(l)}(j)\right|^{2}\right),
\end{aligned}
$$

where

$$
c^{(l)}(j)=\sum_{k \in \Lambda_{n}} \varphi(j, k) u_{l}(k), \quad \mathcal{B}_{n} u_{l}=\lambda_{l} u_{l}
$$

as in the proof of Theorem 1.5. Hence,

$$
\mathcal{T}_{m n} \geq \bigoplus_{l=0}^{n-1}\left(\tilde{\mathcal{A}}+\left(\frac{n}{2 \pi}\right)^{2} \lambda_{l} e^{-2 j / m}+W\right)
$$

and

$$
N\left(\mathcal{T}_{m n} ; 0\right) \leq \sum_{l=0}^{n-1} N\left(\tilde{\mathcal{A}}+\left(\frac{n}{2 \pi}\right)^{2} \lambda_{l} e^{-2 j / m}+W ; 0\right),
$$

where $\tilde{\mathcal{A}}=-m^{2} e^{-2 j / m} \Delta^{2}$. We have that

$$
\begin{aligned}
\sharp\left\{j:\left(\frac{n}{2 \pi}\right)^{2} \lambda_{l} e^{-2 j / m}+W(j)<0\right\} & <\sum_{j \in \mathbb{Z}} \frac{1}{\left(\frac{n}{2 \pi}\right)^{2} \lambda_{l}}\left(-W(j) e^{2 j / m}\right) \\
& =\frac{1}{\left(\frac{n}{2 \pi}\right)^{2} \lambda_{l}}\left\|V_{-}\right\|_{\tilde{Y}} .
\end{aligned}
$$

Lemma 2.1 gives (17).

Remark 3.4.

(i) The inequality following from Lemma 2.1 used in the above proof, namely,

$$
N\left(\tilde{\mathcal{A}}+\left(\frac{n}{2 \pi}\right)^{2} \lambda_{l} e^{-2 j / m}+W ; 0\right) \leq \sharp\left\{j:\left(\frac{n}{2 \pi}\right)^{2} \lambda_{l} e^{-2 j / m}+W(j)<0\right\}
$$


shows that the lower spectrum of the operator $\mathcal{T}_{m n}$ will be finite if

$$
W_{-}(j) \leq\left(\frac{n}{2 \pi}\right)^{2} \lambda_{0} e^{-2 j / m}
$$

for sufficiently large $|j|$. This corresponds to Kneser's theorem [3, ch. XI; 5] for the Sturm-Liouville operator, which states that $-u^{\prime \prime}+q(r) u=\lambda u$ has finite lower spectrum if $q_{-}(r) \leq \frac{1}{4 r^{2}}$ for large $r$; for the radial magnetic Schrödinger equation (with angular momentum quantum number $\tilde{m}=0$ )

$$
-u^{\prime \prime}+\frac{(\tilde{\Psi}+\tilde{m})^{2}-1 / 4}{r^{2}} u+q(r) u=\lambda u
$$

this will be the case if $\frac{\tilde{\Psi}^{2}}{r^{2}}+q(r) \geq 0$.

With $r_{j}=e^{j / m}$ we have the discretised potential $W(j)=q\left(r_{j}\right)$, so our condition is

$$
q_{-}\left(r_{j}\right) \leq\left(\frac{n}{2 \pi}\right)^{2} \lambda_{0} \frac{1}{r_{j}^{2}}=\frac{\tilde{\Psi}^{2}}{r_{j}^{2}}\left(\frac{n}{\pi \tilde{\Psi}} \sin \frac{\tilde{\Psi} \pi}{n}\right)^{2} \sim \frac{\tilde{\Psi}^{2}}{r_{j}^{2}} \quad(n \rightarrow \infty) .
$$

(ii) Similarly, the $l$ th term in (17) is analogous to the Bargmann inequality

$$
\int_{0}^{\infty} q_{-}(r) r d r>2|\tilde{m}+\tilde{\Psi}| N
$$

where $N$ is the number of negative eigenvalues of (18) (cf. [9]); indeed,

$$
\int_{0}^{\infty} q_{-}(r) r d r=\int_{-\infty}^{\infty} q_{-}\left(e^{s}\right) e^{2 s} d s
$$

corresponds to the discrete sum

$$
\frac{1}{m} \sum_{j \in \mathbb{Z}} W_{-}(j) e^{2 j / m}
$$

Acknowledgement. We would like to thank the referee for very helpful comments.

\section{References}

[1] A. A. Balinsky, W. D. Evans, and R. T. Lewis, On the number of negative eigenvalues of Schrödinger operators with an Aharonov-Bohm magnetic field, R. Soc. Lond. Proc. Ser. A Math. Phys. Eng. Sci. 457 (2001), no. 2014, 2481-2489.

[2] M. Cwikel, Weak type estimates for singular values and the number of bound states of Schrödinger operators, Ann. Math. (2) 106 (1977), no. 1, 93-100. 
[3] P. Hartman, Ordinary differential equations, John Wiley \& Sons Inc., New York, 1964.

[4] T. Kato, Perturbation theory for linear operators, 2nd ed., Grundlehren der Mathematischen Wissenschaften, vol. 132, Springer-Verlag, Berlin, 1976; reprinted in Classics in Mathematics, vol. 21, Springer-Verlag, New York, 1980.

[5] A. Kneser, Untersuchungen über die reellen Nullstellen der Integrale linearer Differentialgleichungen, Math. Ann. 42 (1893), no. 3, 409-435.

[6] A. Laptev and T. Weidl, Hardy inequalities for magnetic Dirichlet forms, Mathematical results in quantum mechanics (Prague, 1998), Oper. Theory Adv. Appl., vol. 108, Birkhäuser, Basel, 1999, pp. 299-305.

[7] E. Lieb, Bounds on the eigenvalues of the Laplace and Schroedinger operators, Bull. Amer. Math. Soc. 82 (1976), no. 5, 751-753.

[8] G. V. Rozenbljum, Distribution of the discrete spectrum of singular differential operators, Dokl. Akad. Nauk SSSR 13 (1972), 1012-1015.

[9] K. M. Schmidt, A short proof for Bargmann-type inequalities, R. Soc. Lond. Proc. Ser. A Math. Phys. Eng. Sci. 458 (2002), no. 2027, 2829-2832. 